\section{Ösophaguskarzinom: Wann eignet sich die Endoskopie zur Operation?}

\begin{abstract}
Für Patienten mit einem frühen Adenokarzinom des Ösophagus hat die Lymphknotenbeteiligung große Bedeutung. Daher wurde die Inzidenz von Lymphknotenmetastasen bei diesen Patienten genauer untersucht.
\end{abstract}

Thenting herapie der Wahl bei Patienten mit frühem Adenokarzinom des Ösophagus ist die Ösophagektomie mit Lymphknotendissektion. Allerdings sind für diese belastende Operation vie-

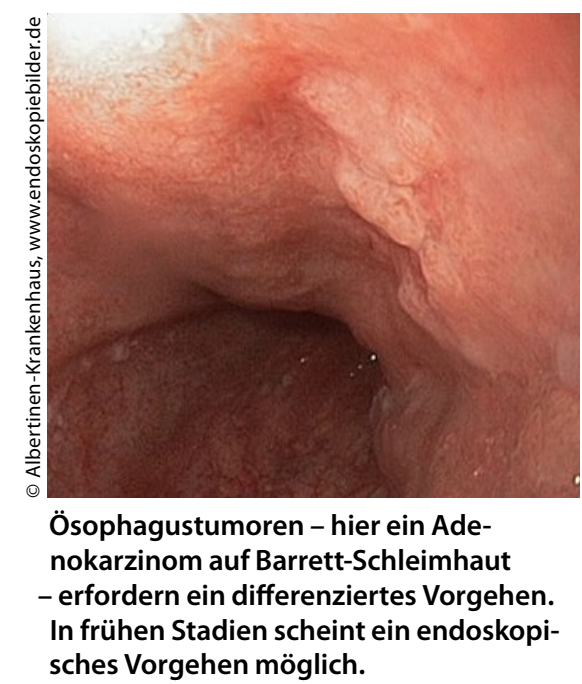

le Patienten wegen hohen Alters oder Komorbiditäten nicht geeignet. Zunehmend wird daher die endoskopische Therapie angewendet, deren Effektivität und Sicherheit in Beobachtungsstudien gezeigt wurden. Die langfristige Mortalität von endoskopischem und offenem Vorgehen ist vergleichbar. Derzeit empfiehlt das National Comprehensive Cancer Network (NCCN) die endoskopische Resektion für Tis-, T1a- und einige T1bLäsionen mit nachfolgender Radiofrequenzablation. Allerdings muss die Lymphknotenbeteiligung gering sein.

Für die aktuelle Untersuchung wurden die Daten von Patienten aus dem SEER(Surveillance, Epidemiology, and End Results)-Register des National Cancer Institutes herangezogen.

Bei 715 Patienten mit Tis-, T1a- und T1b-Tumoren waren in der multivariaten Analyse Tumorgrad, T-Klassifikation und Tumorgröße als unabhängige
Faktoren mit dem Auftreten von Lymphknotenmetastasen assoziiert. Bei „highgrade"-Tumoren war die Wahrscheinlichkeit für eine Lymphknotenbeteiligung höher als bei niedriggradigen (Odds Ratio [OR] 2,76, $\mathrm{p}<0,001$ ), außerdem bei Patienten mit größeren Tumoren $(\geq 2 \mathrm{~cm})$ im Gegensatz zu kleinen Tumoren $(<2 \mathrm{~cm})(\mathrm{OR} 2,68, \mathrm{p}=0,001)$. Patienten mit Tla-Tumoren hatten seltener Lymphknotenmetastasen als Patienten mit Tlb-Tumoren (OR 0,47, $p=0,025)$. Bei Tis-Tumoren war keine Ausbreitung in die Lymphknoten zu beobachten. Bei Patienten mit niedriggradigen (gut oder moderat differenzierten) Tumoren und einer Größe $<2 \mathrm{~cm}$ betrug das Risiko für Lymphknotenmetastasen $1,7 \%$ bei T1a- $(\mathrm{p}<0,001)$ und $8,6 \%$ bei T1b-Tumoren $(\mathrm{p}=0,001)$.

Fazit: Diese Ergebnisse stützen die Empfehlung, bei stark selektierten Patienten mit kleinen Adenokarzinomen des Ösophagus im Frühstadium endoskopisch statt offen zum operieren, wenn konsequentes endoskopisches und radiologisches Monitoring erfolgt. Judith Neumaier

Gamboa AM et al. Treatment Allocation in Patients With Early-Stage Esophageal Adenocarcinoma: Prevalence and Predictors of Lymph Node Involvement. Cancer. 2016;122(14): 2150-7.

\title{
T2-Ösophaguskarzinom: Operation oftmals ausreichend
}

\section{Bei Ösophaguskarzinomen im Stadium T2 besteht ein erhöhtes Risiko für Lymphknotenmetastasen. Untersucht wurde nun, ob eine neoadjuvante Radiochemotherapie vor einer Operation Vorteile hat.}

\footnotetext{
In der Studie wurden die Daten von Patienten verglichen, die vor der Operation eine neoadjuvante Chemo- oder Radiochemotherapie erhielten $(n=70)$, mit denen, die lediglich operiert wurden $(\mathrm{n}=285)$. Durch Propensity Score Matching wurden Baseline-Unterschiede in den Patientencharakteristika ausgeglichen. Nach Adjustierung für Propensity Score und Mangelernährung fanden sich hinsichtlich Klinikmorbidität und -mortalität keine signifikanten Unterschiede zwischen den Therapien. Eine Lymphknotenbeteiligung war bei $50 \%$ der Pati-
}

enten mit alleiniger Operation vorhanden $(20 \%$ pN2/N3). Die neoadjuvante Therapie war mit einem signifikanten Downstaging assoziiert, was sich in der Zunahme der Stadien pT0 (22,9 vs. 2,8\%; p $<0,001)$, pN0 (61,4 vs. $51,9 \%$; $\mathrm{p}=0,041)$ und pTNM0 (18,6 vs. $2,1 \%$; p < 0,001) spiegelte. Sie hatte allerdings keinen signifikanten Effekt auf Gesamtüberleben (OS; 39,2 vs. 43,3 Monate; $\mathrm{p}=0,564$ ) oder krankheitsfreies Überleben (39,2 vs. 40,9 Monate; $p=0,457$ ). Das galt auch für die Subgruppen mit Plattenepithel- oder Adenokarzinom. Auch bei der Entwick- lung von Rezidiven zeigten sich nach 5 Jahren keine signifikanten Unterschiede. Auch waren die Ergebnisse der neoadjuvanten Chemo(radio-)therapie sowohl in der kurz- als auch in der langfristigen Prognose vergleichbar.

Fazit: Die neoadjuvante Therapie hatte für Patienten mit cT2N0-Ösophaguskarzinom keinen Nutzen hinsichtlich des OS. Deshalb sollte diesen Patienten die alleinige operative Behandlung empfohlen werden, auch wenn etwa die Hälfte zu diesem Zeitpunkt bereits eine Lymphknotenbeteiligung hat. Judith Neumaier

Markar SR et al. Role of neoadjuvant treatment in clinical T2N0M0 oesophageal cancer: results from a retrospective multi-center European study. Eur J Cancer. 2016;56:59-68. 